\title{
Evaluation of Oxidative Status in Patients with Brucellosis
}

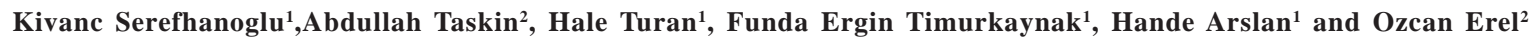 \\ ${ }^{1}$ Baskent University, Medical Faculty, Department of Infectious Diseases and Clinical Microbiology; Ankara,Turkey.; ${ }^{2}$ Harran University, \\ Medical Faculty, Department of Biochemistry; Sanliurfa, Turkey
}

\begin{abstract}
Oxidative stress can be defined as an increase in oxidants and/or a decrease in antioxidant capacity. We aimed to determine total antioxidant capacity (TAC), total peroxide, malondialdehyde and catalase levels in plasma samples, and calculation of oxidative stress index (OSI) in patients with brucellosis to evaluate their oxidative status using a novel automated method. Sixty-nine patients with brucellosis and 69 healthy control subjects were included in the present study. Plasma levels of total peroxide and malondialdehyde were significantly increased in patients as compared with healthy controls $(\mathbf{p}<\mathbf{0 . 0 0 1}$ and $\mathbf{p}<\mathbf{0 . 0 0 1}$, respectively). In contrast, TAC level was significantly lower in patients as compared with controls $(\mathbf{p}<\mathbf{0 . 0 0 1})$. There was no statistically significant difference between the catalase results of the two groups $(p>0.05)$. OSI level was significantly increased in patients as compared with healthy controls $(\mathbf{p}<\mathbf{0 . 0 0 1})$. In conclusion, oxidants were increased and antioxidants were decreased in patients with brucellosis. Oxidative stress was increased in patients with brucellosis.
\end{abstract}

Key-Words: Brucellosis, oxidants, antioxidants, oxidative stress.

Numerous studies demonstrated that in the many infectious diseases, a variety of inflammatory cells are activated, which lead to production of reactive oxygen and nitrogen species to kill intra-cellular and extra-cellular parasites $[1,2]$. Reactive oxygen species are one of the crucial molecules that kill bacteria internalized into phagocytic cells, such as polymorphonuclear neutrophils (PMNs) and macrophages [3]. They reside in the phagocytic cells and are released into the phagosome upon engulfment of bacteria [4]. These molecules fight against bacteria by causing DNA strand breaks, degradation of RNA, inhibition of amino acid biosynthesis, and inactivation of membrane transport proteins [5].

Brucella is a Gram-negative, facultative, intracellular pathogen that produces cell toxicity by altering plasma membrane and inducing cell apoptosis [6]. It is stated that the oxidative killing pathways of host macrophages represent a primary mechanism utilized by these host phagocytes to control the intracellular replication of the brucellae [7]. Therefore, it is possible that brucellosis may be related to increased free radical production and antioxidant depletion, and oxidative stress may be implicated in the pathogenesis of brucellosis. Thus, in the present study, we evaluated the oxidative status in patients with brucellosis and healthy controls via measurement of total antioxidant capacity (TAC), oxidants (total peroxide and malondialdehyde-a lipid peroxidation byproduct) and antioxidant (catalase) levels in plasma samples, and calculation of oxidative stress index (OSI).

\section{Material and Methods}

The study was conducted at Baskent Univercity, Konya

Received on 14 April 2009; revised 7 July 2009.

Address for correspondence: Dr. Kivanc Serefhanoglu. Baskent University, Medical Faculty, Department of Infectious Diseases and Clinical Microbiology, 06490-Ankara,Turkey. Tel.: +90 216 5541500, Fax: 902166519858.

The Brazilian Journal of Infectious Diseases 2009;13(4):249-251. (C) 2009 by The Brazilian Journal of Infectious Diseases and Contexto Publishing. All rights reserved.
Medical and Research Center in Konya, Turkey. Informed consents were obtained and the study protocol was approved by the Ethical Committee of the Baskent University Hospital. Diagnosis of brucellosis was made by isolation of the bacterium from blood sample and/or serum agglutination test with titers $\geq 1: 160$ in conjunction with a compatible clinical presentation [8].

After overnight fasting, peripheral venous blood sample was collected into heparinized tubes for each individual in the study and control groups, and plasma was separated by centrifugation at $1500 \mathrm{x} g$ for $10 \mathrm{~min}$, and the sample was then stored at $-80^{\circ} \mathrm{C}$ until further analysis of catalase, TAC, total peroxide and malondialdehyde. The blood samples were collected from patients after brucellosis was identified. In the control group, the samples were collected from healty volunteers. At least two blood cultures were also drawn from each patient at diagnosis.

Catalase activity was measured using hydrogen peroxide $\left(\mathrm{H}_{2} \mathrm{O}_{2}\right)$ as substrate [9]. The disappearance of $\mathrm{H}_{2} \mathrm{O}_{2}$ was followed at $240 \mathrm{~nm}$, and enzyme activity was shown as $\mathrm{k} / \mathrm{mg}$ protein ( $k$ : rate constant of a first-order reaction) at $25^{\circ} \mathrm{C}$. The total antioxidant status of the plasma was measured using a novel automated colorimetric measurement method for TAC developed by Erel [10]. The novel automated method is based on the bleaching of characteristic color of a more stable ABTS [2,2'-Azino-bis(3-ethylbenzothiazoline-6-sulfonic acid)] radical cation by antioxidants [11]. The assay has excellent precision values, which are lower than $3 \%$. The results were expressed as mmol Trolox equivalent/L. Total peroxide concentrations of plasma were determined by using the FOX2 method with minor modifications [12,13]. The FOX2 test system is based on the oxidation of ferrous iron to ferric iron by the various types of peroxides contained in the plasma samples in the presence of xylenol orange which produces a colored ferric-xylenol orange complex whose absorbance can be measured. The percent ratio of the total peroxide to the TAC gave the OSI, an indicator of the degree of oxidative stress [13]. Collected data were subjected to statistical analysis using 
SPSS statistical package version 13.0. The data obtained from the study and control groups were compared using Student's t-test and chi-square test. Differences were considered statistically significant at $\mathrm{p}<0.05$.

\section{Results}

Sixty-nine patients with brucellosis and 69 healthy control subjects were included in the present study. Mean age of patients and controls was 45,2 $\pm 19,03$ years and 43,5 $\pm 16,6$ years, respectively ( $\mathrm{p}>0.05)$. Male-to-female ratio of patients and controls was 35/34 and 32/37, respectively ( $\mathrm{p}>0.05$ ). In 63 (91\%) patients, at least one risk factor for brucellosis was identified: raw milk consumption (90\%), close contact with animals (20\%), home slaughtering of sheep or cattle (4\%), or work in an abattoir (3\%). Symptoms of patients were arthralgia (91\%), sweating (87\%), loss of appetite (86\%), fever (83\%), headache (36\%), chills (26\%), and abdominal pain (4\%). Elevated erythrocyte sedimentation rate was determined in $94 \%$, leukopenia in $30 \%$ and anaemia in $27 \%$ of the patients. Blood cultures were positives in $22 \%$ of cases.

As seen in Table 1, plasma levels of total peroxide and malondialdehyde were significantly increased in patients as compared with healthy controls $(\mathrm{p}<0.001$ and $\mathrm{p}<0.001$, respectively). In contrast, TAC level was significantly lower in patients as compared with controls ( $p<0.001)$. There was no statistically significant difference between the catalase results of the two groups ( $\mathrm{p}>0.05)$. OSI level was significantly increased in patients as compared with healty controls $(\mathrm{p}<0.001)$.

\section{Discussion}

In order to investigate the effect of brucellosis on oxidative status, we measured total antioxidant capacity (TAC), oxidative stress index, the oxidants (malondialdehyde and total peroxide) and the antioxidant (catalase). To the best of our knowledge, there is no prior study investigating these biomarkers of oxidation and antioxidant defense in patients with brucellosis. Reactive oxygen species (ROS) are produced during many

Table 1. Plasma oxidative and antioxidative parameters of patients with brucellosis and healty controls.

\begin{tabular}{lccc}
\hline Patients (n=69) & Healty & $\begin{array}{c}\text { Controls } \\
(\mathbf{n}=69)\end{array}$ & p \\
\hline Catalase (k/mg prot) & $25.41 \pm 35.77$ & $26.52 \pm 47.52$ & 0.877 \\
$\begin{array}{l}\text { TAC } \\
\text { (mmol Trolox Equiv/L) }\end{array}$ & $0.62 \pm 0.30$ & $1,08 \pm 0,29$ & $<0.001$ \\
$\begin{array}{l}\text { Total peroxide } \\
\left(\mu \mathrm{mol} \mathrm{H}_{2} \mathrm{O}_{2} / \mathrm{L}\right)\end{array}$ & $38.21 \pm 11.61$ & $21.64 \pm 7.75$ & $<0.001$ \\
$\begin{array}{l}\text { OSI } \\
\text { (arbitrary unit) }\end{array}$ & $85.54 \pm 69.03$ & $21.38 \pm 9.36$ & $<0.001$ \\
$\begin{array}{l}\text { Malondialdeyde } \\
\text { (mmol/L) }\end{array}$ & $12.36 \pm 4.75$ & $5.91 \pm 4.08$ & $<0.001$ \\
\hline
\end{tabular}

Values are mean \pm SD, TAC: total antioxidant capacity; OSI: oxidative stress index. metabolic and physiological processes. Organisms have several enzymatic and non-enzymatic antioxidant systems that overwhelm harmful effects of these ROS. Under certain conditions, antioxidants mechanisms are impaired and/or ROS are increased and antioxidant mechanisms may become insufficient to prevent oxidative damage completely. Consequently, oxidative stress develops [14,15].

Although the level of plasma antioxidant components can be measured separately, these measurements are time consuming, costly, unpractical and labour intensive [11]. It is known that, various antioxidants in plasma have additive effects on oxidative status, and the cooperation of the antioxidants prevents adverse effects of free radicals [16]. Measurement of individual antioxidant components may not accurately reflect the total antioxidant status [11], and measurement of TAC practically represents all of them and therefore can reflect the oxidative status of the organism $[10,17]$. Various measurement methods have been developed to measure total antioxidant status, but there is not yet an accepted reference method. We used a novel automated colorimetric measurement method for TAC developed by Erel [10]. The measurement of TAC by this assay is sensitive, reliable and specific [10]. In our study, we found that TAC level was significantly decreased in the patients with brucellosis compared to the healthy controls, indicating that the anti-oxidative defense of these patients was impaired. A possible mechanism for the decreased level of TAC could be that the radical-scavenging antioxidants were consumed by the increased free radical activity associated with Brucella infection. This opinion was supported by the fact that in the current study, in the patients with brucellosis, significant increases in the individual oxidants, total peroxide and malondialdehde, were detected compared to control subjects. Malondialdehde is produced from lipid peroxidation, and is one of the oxidative stress markers in plasma [18].

In the present study OSI was significantly higher in the patients than controls. OSI is the ratio of the total plasma peroxide level to TAC, and is an indicator of oxidative stress. It has been suggested that OSI may reflect the state of oxidative status more accurately than TAC $[13,19]$.

In the current study, catalase level showed no difference between the patients and controls. Catalase serves as an intracellular antioxidant enzyme, and is a member of free radical and ROS scavenging system [20,21]. Melek et al. [22] using a rat model investigated the activity of catalase and malondialdehde in Brucella infection. Similar to our findins, in these mice, while Brucella infection significantly increased malondialdehde concentration in the plasma, brain, liver, and spleen, the pathogen did not affect catalase activities in any of specimens apart from in liver. In this study, catalase activity in the liver was increased at 30th day of post-infection and then declined to basal level at 45th day.

The oxidative killing by polymorphonuclear leukocytes and macrophages plays a primary role in the elimination of intracellular brucellae. The organism has ability to resist the 
oxidative killing by several incompletely understood mechanisms and thereby can survive and multiply in the these phagocytic cells of the host [23]. Consequently, the ongoing inflammation characterized by activated neutrophils and macrophages is possibly associated to the high production of reactive oxygen species, as we have observed in the present study.

While there is no any report in the literature about TAC, OSI, total peroxide, malondialdehde and catalase in patients with brucellosis, in the several studies, similar to our findings increase in OSI, total peroxide and MDA and/or decrease in TAC have been reported in subjects with several conditions, including chronic hepatitis B infection [24], preeclampsia [25], exposure to passive smoking [26], cutaneous leishmaniasis [27], and chronic renal failure [21]. Ece et al. [21] found significantly lower catalase level in the children with chronic renal failure compared with the control group.

In conclusion, according to the data obtained from the present study, decreased TAC levels, and increased malondialdehde and total peroxide levels together with increased OSÝ showed that the patients with brucellosis infection were exposed to potent oxidative stress.

\section{References}

1. Gantt KR, Goldman TL, McCormick ML, et al. Oxidative response of human and murine macrophages during phagocytosis of Leishmania chagasi. J Immunol 2001;167:893-901.

2. Murray HW, Teitelbaum RF. l-arginine-dependent reactive nitrogen intermediates and the antimicrobial effect of activated human mononuclear phagocytes. J. Infect. Dis 1992;165:513-7.

3. Dieffenbach CW, Tramont EC. Innate (general or nonspecific) host defense mechanisms. In: Mandell GL, Bennett JE, Dolin R (eds). Principles and Practice of Infectious Diseases. Sixth edition. Elsevier Churchill Livingstone, Philadelphia, 34-42, 2005.

4. Rada B, Hably C, Meczner A, et al. Role of Nox2 in elimination of microorganisms. Semin Immunopathol 2008;30:237-53.

5. Park MK, Myers RA, Marzella L. Oxygen tensions and infections: modulation of microbial growth, activity of antimicrobial agents, and immunologic responses. Clin Infect Dis 1992;14:720-40.

6. de Bagu"e's MPJ, Terraza A, Antoine Gross, Dornand J. Different Responses of Macrophages to Smooth and Rough Brucella spp.: Relationship to Virulence. Infection and Immunity. 2004;72:2429-33.

7. Jiang X, Baldwin CL. Macrophage control of Brucella abortus: Role of reactive oxygen intermediates and nitric oxide. Cell Immunol 1993;151:309-19.

8. Pappas G, Akritidis N, Bosilkovski M, Tsianos E. Brucellosis. N Engl J Med 2005;352:2325-36.
9. Aebi H. Catalase. In: H.U. Bergmeyer (ed). Methods of Enzymatic Analysis. Academic Pres Inc., New York and London, 673-7, 1974.

10. Erel O. A novel automated direct measurement method for total antioxidant capacity using a new generation, more stable ABTS radical cation. Clin Biochem 2004;37:277-85.

11. Cao G, Prior RL. Comparison of different analytical methods for assessing total antioxidant capacity of human serum. Clin. Chem 1998;44:1309-15.

12. Yeni G, Gulum M, Selek S, et al. Comparison of oxidative/ antioxidative stasus of penil corpus cavernosum blood and peripheral venous blood. Int J Impot Res 2005;17:19-22.

13. Harma M, Harma M, Erel O. Increased oxidative stress in patients with hydatidiform mole. Swiss Med. Wkly 2003;133:563-6.

14. Halliwell B, Gutteridge JMC. Free Radicals in Biology and Medicine. 3. Oxford; Oxford Science Publications; 1999.

15. Aruoma OI. Characterization of drugs as antioxidant prophylactics. Free Radic Biol Med 1996;20:675-705.

16. Wayner DD, Burton GW, Ingold KU, et al. The relative contributions of vitamin $\mathrm{E}$, urate, ascorbate and proteins to the total peroxyl radical-trapping antioxidant activity of human blood plasma. Biochim Biophys Acta. 1987;924:408-19.

17. Erel O. A novel automated method to measure total antioxidant response against potent free radical reactions. Clin Biochem 2004;37:112-9.

18. Sattler W, Malle E, Kostner GM. Methodological approaches for assessing lipid and protein oxidation and modification in plasma and isolated lipoproteins. Methods Mol Biol 1998;110:67-91.

19. Harma M, Harma M, Erel O. Oxidative stress in women with preeclampsia. Am J Obstet Gynecol. 2005;192:656-7.

20. Aslan M, Kosecik M, Horoz M, et al. Assessment of paraoxonase and arylesterase activities in patients with iron deficiency anemia. Atherosclerosis 2006.

21. Ece A, Gürkan F, Kervancýoðlu M, et al. Oxidative stress, inflammation and early cardiovascular damage in children with chronic renal failure. Pediatr Nephrol 2006;21:545-52.

22. Melek IM, Erdogan S, Celik S, et al. Evaluation of oxidative stress and inflammation in long term Brucella melitensis infection. Molecular and Cellular Biochemistry 2006;293:203-9.

23. Young EJ. Brucella species. In: Mandell GL, Bennett JE, Dolin R (Eds). Principles and Practice of Infectious Diseases, Sixth edition. Elsevier Churchill Livingstone, Philadelphia, 2669-74, 2005

24. Bolukbas C, Filiz Bolukbas F, Horoz M, et al. Increased oxidative stress associated with the severity of the liver disease in various forms of hepatitis B virus infection. BMC Infect Dis 2005;5:95.

25. Harma M, Harma Muge, Erel O. Measurement of the total antioxidant response in preeclampsia with a novel automated method. European Journal of Obstetrics \& Gynecology and Reproductive Biology 2005;118:47-51.

26. Kosecika M, Erel O, Sevinc E, Selek S. Increased oxidative stress in children exposed to passive smoking. International Journal of Cardiology 2005;100:61-4.

27. Kocyigit A, Keles H, Selek S, et al. Increased DNA damage and oxidative stress in patients with cutaneous leishmaniasis. Mutation Research 2005;585:71-8. 